\title{
Perfect One-Factorization Conjecture
}

\author{
Chriestie E. J. C. Montolalu ${ }^{1}$ \\ ${ }^{1}$ Program Studi Matematika, FMIPA, UNSRAT Manado, chriestelly@yahoo.com
}

\begin{abstract}
Perfect one-factorization of the complete graph $K_{2 n}$ for all $n$ greater and equal to 2 is conjectured. Nevertheless some families of complete graphs were found to have perfect one-factorization. This paper will show some of the perfect one-factorization results in some families of complete graph as well as some result in application.
\end{abstract}

Keywords: complete graph, one-factorization

\section{Dugaan Perfect One-Factorization}

\begin{abstract}
Abstrak
Perfect one-factorization pada graph lengkap $K_{2 n}$ untuk semua $n$ lebih dari dan sama dengan 2 masih diduga. Namun beberapa graph lengkap dibuktikan memiliki perfect one-factorization. Tulisan ini akan menunjukkan beberapa perfect one-factorization yang ada pada beberapa graph dan juga beberapa hasil dalam aplikasinya.
\end{abstract}

Kata kunci: graph lengkap, one-factorization

\section{Introduction}

The existence of a perfect one-factorization of the complete graph $K_{2 n}$ for all $n \geq 2$ is conjectured. This has been suggested by Kotzig in 1964 in his paper "Hamiltonian Graphs and Hamiltonian Circuits" [1].

Three families of perfect one-factorization are known to be exist. The first family was proven by Kotzig himself. In his paper [1], Kotzig proved that there exists a perfect onefactorization of the complete graph $K_{p+1}$ for all odd primes $p$. The second family was constructed by Anderson in 1973 [2], i.e. the perfect one-factorization for complete graph $K_{2 p}$ exists for all odd prime $p$. This was also constructed independently by Nakamura in 1975 [3]. Nevertheless, these two results were proven to be isomorphic by Kobayashi [4] in 1989. Long after that, in 2006, a new family which is the third family was shown in [5]. Bryant, Maenhaut and Wanless constructed a perfect one-factorization of $K_{p+1}$, for each prime $p \geq 11$ which is not isomorphic to complete graph in the first family. These three families have become reference for the studies in perfect onefactorization, especially for the work of proving the existence of perfect one-factorization for all complete graph $K_{2 n}$.

This essay outlines some studies in perfect one-factorization conjecture as well as the result of some complete graphs that have been shown to have perfect one-factorization and some brief applications of perfect one-factorization.

\section{Perfect One-Factorization}

For any graph $G$, a factor or a spanning subgraph of $G$ is a subgraph with vertex-set $V(G)$. A factorization of $G$ is a set of factors of $G$ that are pairwise edge-disjoint (no two have a common edge) and whose union is all of $G$. In particular, a one-factor is a factor that is a regular graph of degree 1 , in other words, a one-factor is a set of pairwise disjoint edges of $G$ that between them contain every vertex. A one-factorization of $G$ is a decomposition of the edge-set of $G$ into edge disjoint one-factors. 
Example 1. A graph with one-factor and one-factorization in Desargues graph

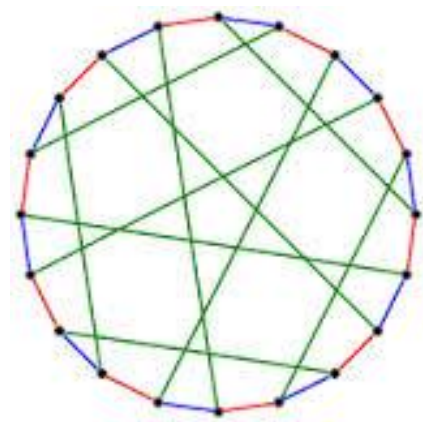

Figure 1. Desargues graph

There are three one-factors in the picture edge-colored by three different colors (red, blue, green). The union of the three factors forms the graph, thus this is an example of a graph that has onefactorization.

A perfect one-factorization of $G$ is a one-factorization in which the union of any pair of onefactors is a Hamiltonian cycle of $G$. Consider the graph in the example above, the union of the red one-factor and the blue-one factor induces a Hamiltonian cycle. Furthermore, it is also can be seen that both the union of the red and green and the blue and green induce Hamiltonian cycles in the graph. Therefore, that graph is also an example of a graph with perfect one-factorization.

\section{Some Studies of Perfect One-Factorization Conjecture}

\subsection{Perfect Pair of One-Factorization and Perfect Near One-Factorization}

In his paper in 1989, Wagner [6] proved the lower bound of maximum perfect pairs of onefactorization in a complete graph. This was shown by first considering a graph $G$ that has a onefactorization $\mathscr{F}$ with set of one-factors $\mathscr{T}=\left\{F_{1}, \ldots, F_{d}\right\}$. The perfect pair of $\mathscr{T}$ is a pair $\left\{F_{k}, F_{l}\right\}$ such that $F_{k} \cup F_{l}$ induces a Hamiltonian cycle in $G$.

Let $c(\mathscr{N})$ to be the number of perfect pairs of $\mathscr{F}$, and $c(G)$ to be the maximum $c(\mathscr{T})$ over all one-factorizations $\mathscr{F}$ of $G$. Since the conjecture is the existence of a perfect one-factorization of the complete graph $K_{2 n}$ for all $n \geq 2$, it can be assumed that a one-factorization of $K_{2 n}$ exists in which every pair is perfect. Wallis in [7] proved in theorem 6.3. that if $v$ is even, then $K_{v}$ can be factored into $\frac{v}{2}-1$ Hamilton cycles and a one factor. This implies that there can be $2 n-1$ onefactors in a $K_{2 n}$. Thus if $K_{2 n}$ has a one-factorization $\mathscr{F}$ with the set of one-factors $\mathscr{F}=\left\{F_{1}, \ldots, F_{d}\right\}$ , then $d=2 n-1$. Then, the perfect one-factorization conjecture for $n \geq 2$ satisfies

$$
c\left(K_{2 n}\right)=\left(\begin{array}{c}
2 n-1 \\
2
\end{array}\right) \text {. }
$$

The related problem in this case is to find a set of $\left(\begin{array}{c}2 n-1 \\ 2\end{array}\right)$ Hamilton cycles in $K_{2 n}$ such that each edge in $K_{2 n}$ is on exactly one of the cycles.

Furthermore, in this paper perfect near-one-factorization is also considered to be useful in determining the conjecture. It is well known that $K_{2 n}$ has a perfect one-factorization if and only if $K_{2 n-1}$ has a perfect near one-factorization. Recall that a near-one-factor of $G=(V, E)$ is a onefactor of $G \backslash v$ for some $v \in V$ and a near-one-factorization of $G$ is a partition $\mathscr{F}=\left\{F_{1}, \ldots, F_{S}\right\}$ of $E$ into near-one-factors. A pair $\left\{F_{k}, F_{l}\right\}$ in $\mathscr{F}$ is a perfect pair if $F_{k} \cup F_{l}$ induces a Hamiltonian path in $G$. A near-one-factorization is perfect if and only if all of its pairs are perfect.

For odd $2 n-1, c\left(K_{2 n}\right)=\left(\begin{array}{c}2 n-1 \\ 2\end{array}\right)$ if $n$ is prime or $2 n-1$ is prime. This leads to the families of complete graph with perfect one-factorization. Based on these, the lower bound for the maximum of perfect pair in complete graph of order $2 n$ can be obtained as:

$$
c\left(K_{2 n}\right) \geq(2 n-1) \cdot \varphi(2 n-1) / 2 \text {, where } \varphi \text { is the Euler totient function }
$$

Moreover, by considering this inequality, another inequality can be obtained as follows:

If $m$ and $n$ are odd and coprime then

$$
c\left(K_{m n}\right) \geq 2 . c\left(K_{m}\right) \cdot c\left(K_{n}\right)
$$


Since $m n$ is odd which means $m n+1$ is even, then regarding the perfect near-one-factorization properties, one can obtain:

$$
\begin{aligned}
& \text { If } m \text { and } n \text { are odd and coprime then } \\
& c\left(K_{m n+1}\right) \geq 2 . c\left(K_{m+1}\right) . c\left(K_{n+1}\right)
\end{aligned}
$$

These inequalities can lead to assumption that if the maximum number of perfect pairs in onefactorization set of complete graph of even number can be obtained, at least an estimated number, then there may exist perfect pairs for all one-factorizations of complete graph of even number, $K_{2 n}$ for all $n \geq 2$. Therefore, further study for the conjecture which is based on these results can be conducted.

\section{2. $\quad 2^{\alpha-1}-q u o t i e n t$ Starters In Finite Fields}

Dinitz and Stinson [8] constructed seven examples of perfect one-factorizations by using twoand four-quotient starters in finite fields. A starter in an additive abelian group $G$ of order $2 n-1$ is a set $S=\left\{\left\{x_{1}, y_{1}\right\},\left\{x_{2}, y_{2}\right\}, \ldots,\left\{x_{n-1}, y_{n-1}\right\}\right\}$ such that every non-zero element of $\mathrm{G}$ occurs as

(1) an element in exactly one pair of $S$, and

(2) a difference of exactly one pair of $S$.

For example, $\{\{1,6\},\{2,5\},\{3,4\}\}$ is a starter in $Z_{7}$.

Let $S^{*}=S \cup\{0, \infty\}$. For any $g \in G$, define

$$
\begin{gathered}
S^{*}+g=\left\{\left\{x_{1}+g, y_{1}+g\right\},\left\{x_{2}+g, y_{2}+g\right\}, \ldots,\left\{x_{n-1}+g, y_{n-1}+g\right\}\right\}, \\
\text { where } \infty+g=g+\infty=\infty \text { for all } g \in G
\end{gathered}
$$

Then, it is clear that $F=\left\{S^{*}+g\right\}$ for all $g \in G$ is a one-factorization of $K_{2 n}$. To check the perfect properties of this, instead of checking all $\left(\begin{array}{c}2 n-1 \\ 2\end{array}\right)$ pairs of one-factors, choose $n-1$ nonzero group elements $g_{1}, g_{2}, \ldots, g_{n-1}$ such that no two of them sum to zero. Then, $F$ is perfect if and only if $S^{*} \cup\left(S^{*}+g_{i}\right)$ is a Hamiltonian cycle for $1 \leq i \leq n-1$.

For example $S^{*}=\{\{1,6\},\{2,5\},\{3,4\},\{0, \infty\}\}$.

Consider $G=\{0,1,2,3,4,5,6\}$

Then for all $g \in G, S^{*}+g$ will give an orbit in $K_{8}$ as follows:

$S^{*}+g=\{\{1,6\},\{2,5\},\{3,4\},\{0, \infty\}$,

$\{2,0\},\{3,6\},\{4,5\},\{1, \infty\}$,

$\{3,1\},\{4,0\},\{5,6\},\{2, \infty\}$,

$\{4,2\},\{5,1\},\{6,0\},\{3, \infty\}$

$\{5,3\},\{6,2\},\{0,1\},\{4, \infty\}$

$\{6,4\},\{0,3\},\{1,2\},\{5, \infty\}$,

$\{0,5\},\{1,4\},\{2,3\},\{6, \infty\}\}$

$S^{*}+g$ is a one-factorization of $K_{8}$ with vertex set $V=\{0,1,2,3,4,5,6, \infty\}$.

Check the perfect properties as follows,

Suppose two distinct groups in which no two elements on each of them sum to zero.

Consider $G_{1}=\{1,2,3\}$ and $G_{2}=\{4,5,6\}$

then it is clear that for each $g_{i} \in G_{1}$ or $G_{2}, S^{*} \cup\left(S^{*}+g_{i}\right)$ is a Hamiltonian cycle. Thus, $F=\left\{S^{*}+g\right\}$ is perfect.

Suppose $q=2^{\alpha} t+1$ is an odd prime power, where $t$ is odd.

Let $\omega$ be a primitive element in Galois field $G F(q)$, and let $C_{0}$ be the unique subgroup of $G^{*}$ of order $t$ and index $2^{\alpha}$, where $G^{*}$ denotes the multiplicative group $G \backslash\{0\}$.

Denote the coset of $C_{0}$ by $C_{i}, 0 \leq i \leq 2^{\alpha}-1$, where $C_{i}=\omega^{i} C_{0}$.

For example, let us consider $G F(13)$.

Since $13=2^{2} .3+1$, the sub-group $C_{i}$ is of order 3 and there are $2^{2}=4$ subgroups

In multiplicative group $G \backslash\{0\}$, the set of coprime number to 13 is

$$
G^{*}=\{1,2,3, \ldots, 12\}
$$

Choose a cyclic subgroup generated by 3 , i.e. $3^{0}=1,3^{1}=3$, and $3^{2}=9$, then

$C_{0}=\{1,3,9\}$, and the other cosets can be derived as follows:

$C_{1}=\{2,6,5\}, C_{2}=\{4,12,10\}$, and $C_{3}=\{7,8,11\}$. 
A starter $S$ in $G F(q)$ is said to be a $2^{\alpha-1}$-quotient coset starter if the following property is satisfied: for all pairs $\{x, y\},\left\{x^{\prime}, y^{\prime}\right\} \in S$ if $x, x^{\prime} \in C_{i}$ for some $i$, then $\frac{y}{x}=\frac{y \prime}{x^{\prime}}$

For example, in polynomial form, consider $q=25=5^{2}=2^{3} 8+1$. $G F(25)$ can be constructed from the irreducible polynomial $x^{2}+x+2$ over $G F(5)$. Then $x$ is a primitive element. By calculating a subgroup with a generator, it can be found that $C_{0}=\left(x^{1}, x^{13}, x^{10}, x^{14}\right)$. This can be a starter in form $S\left(x^{1}, x^{13}, x^{10}, x^{14}\right)$ and it is clear that it is a 4-quotient coset starter.

By using this fact, Dinitz and Stinson proved that a starter $S$, where $S$ is a $2^{\alpha-1}$-quotient coset starter in $G F(q)$, generates a perfect one-factorization if and only if $S^{*} \cup\left(S^{*}+g_{i}\right)$ is a Hamiltonian cycle for $1 \leq i \leq 2^{\alpha-1}-1$.

For example consider again the previous example for $q=25$. The starter $S\left(x^{1}, x^{13}, x^{10}, x^{14}\right)$ in $G F(25)$ generates perfect one-factorization. This can be done by checking that both $S^{*} U$ $\left(S^{*}+x\right)$ and $S^{*} \cup\left(S^{*}+x^{2}\right)$ induce Hamiltonian cycle.

By using this method, perfect one-factorization of complete graph $K_{2 n}$ can be obtained.

For example, consider a complete graph of order $126, K_{126}$.

Then take $q=125=5^{3}=2^{2} 31+1$.

Construct $G F\left(5^{3}\right)$ from the polynomial $x^{3}+x^{2}+2$, which is irreducible over $Z_{5}$. Then, $x$ is a generator. $S\left(x^{9}, x^{41}\right)=S\left(2 x^{2}+4 x+4, x^{2}+2 x+3\right)$ generates a perfect one-factorisation of $K_{126}$.

\section{Uniform One-Factorization}

Another approach to perfect one-factorization is by considering the uniform of onefactorization. This was used by Dinitz and Dukes in [9] regarding the cycle structure of onefactorization.

A one factorization $\left\{F_{1}, \ldots, F_{2 n-1}\right\}$ of $K_{2 n}$ is uniform if the graphs with edge sets $\quad F_{i} \cup F_{j}$ are all isomorphic for $i \neq j$. Since the union of two one-factors is a 2-regular graph, then it is isomorphic to a disjoint union of even cycles. If $F_{i} \cup F_{j}$ is isomorphic to the disjoint union of cycles of lengths $k_{1}, \ldots, k_{r}$, then the multiset $T=\left(k_{1}, \ldots, k_{r}\right)$ is said to be the type of a uniform onefactorization. In other words, a uniform one-factorization "contains a $k$-cycle" if $k$ occurs in its type. A one-factorization of $K_{2 n}$ is perfect if it is uniform of type $2 n$.

In this paper, Dinitz and Dukes show that for each even $k>4$ and any positive integer $N$ there exists a uniform one-factorization in some large enough complete graph containing at least $N$ number of $k$-cycles. This means that, with the knowledge of $2 n$-cycle in a complete graph, there can be a possibility for a large complete graph of order $2 n$ for all $n$ positive integer to have perfect one-factorizations.

\section{Some Constructed Results}

1974, Anderson and Morse [10] constructed perfect one-factorizations of $K_{16}$ and $K_{28}$

1988, Seah and Stinson [11] constructed perfect one-factorizations of $K_{36}$

1989, Seah and Stinson [12] constructed perfect one-factorizations of $K_{40}$

1987, Ihrig, Seah, and Stinson [13] constructed perfect one-factorizations of $K_{50}$

2009, Wolfe constructed perfect one-factorizations of $K_{52}$

1988, Kobayashi and Kiyasu-Zen'iti [14] constructed perfect one-factorizations of $K_{1332}$ and $K_{6860}$ By using 2- and 4-quotient starters in finite fields, Dinitz and Stinson [9] constructed $K_{126}, K_{170}$, $K_{730}, K_{170}, K_{1370}, K_{1850}, K_{2198}$, and $K_{3126}$.

Wanless in webpage [15] listed the known existence results of a perfect one-factorization of $K_{n+1}$ exists if $n$ is odd and

- $n<50$

- $n=p$ for some prime $p$ (two non-isomorphic constructions known),

- $n=2 p-1$ for some prime $p$

- $n=p^{2}$ for some prime $p<280$, such that $p=3$ or $5 \bmod 8$

- $n=p^{2}$ for some prime $p<30$, such that $p=7 \bmod 8$

- $n=p^{3}$ for some prime $p<300$, such that $p=3 \bmod 4$ 
- $n=p^{3}$ for some prime $p<150$, such that $p=5 \bmod 8$

- $n=p^{4}$ for some prime $p<6$

- $n=p^{5}$ for some prime $p<25$ except possibly $p=17$

- $n=p^{6}$ for some prime $p<6$

- $n=5^{7}$

\section{Some Applications}

\subsection{Shortening array codes}

Array codes are erasure-correcting codes represented by an array bits. Erasures correspond to the loss of columns. A two erasure correcting array code, for example, is capable of recovering any two lost columns.

For example, a simple array codes A simple two-erasure correcting array code of length four is shown in table 1.

Table 1. Array code

\begin{tabular}{|r|r|r|r|}
\hline$a$ & $b$ & $c$ & $d$ \\
\hline$b+c$ & $c+d$ & $d+a$ & $a+b$ \\
\hline
\end{tabular}

The first row consists of four information bits $a, b, c$, and $d$.

The second row contains four parity bits.

The '+' sign indicates bitwise exclusive-OR, so that $x+x=0$.

Suppose, for example, that columns three and four are lost (as shown in Table 2). Table 2. Lost array code

\begin{tabular}{|r|r|r|r|}
\hline$a$ & $b$ & & \\
\hline$b+c$ & $c+d$ & & \\
\hline
\end{tabular}

$c$ can be recovered by adding $b+c$ to $b: c=(b+c)+b$

$d$ can be recovered by using $c+d$ and $c: d=(c+d)+c$

The $B$-Code is a two-erasure correcting array code of length $2 n$, represented by a $n$ by $2 n$ array. It can recover any two out of $2 n$ lost columns. The construction of the B-Code is based on the perfect one-factorization of the complete graph, $K_{2 n}$.

In [16], Bohossian and Bruck used the constructed perfect one-factorization to shorten arbitrary array codes. Moreover, these codes can be used to derive a new family of the conjecture. The derivation they used is as follows:

(1) Find an existed perfect one-factorization of $K_{2 n}$,

(2) Construct extended B-Code of length $2 n-1, B_{2 n-1}$, from $K_{2 n}$ in (1)

(3) Generalize X-Code of length $n, \tilde{X}_{n}$ by shortening new construction from $B_{2 n-1}$,

(4) Construct extended B-Code of length $n, B_{n}$, from $\tilde{X}_{n}$ by separation

(5) Construct a new perfect one-factorization of $K_{n+1}$ from $B_{n}$.

Above derivation can be done in an example as follows:

Consider $K_{10}$ which is known to have a perfect one-factorization with one-factors that can be listed as shown in Table 3.

Table 3. One-factors of a perfect one-factorization

\begin{tabular}{|c|c|c|c|c|c|c|c|c|}
\hline$f_{5}$ & $f_{2}$ & $f_{4}$ & $f_{6}$ & $f_{8}$ & $f_{1}$ & $f_{3}$ & $f_{7}$ & $f_{9}$ \\
\hline$(0,5)$ & $(1,6)$ & $(2,7)$ & $(3,8)$ & $(4,9)$ & $(0,1)$ & $(0,3)$ & $(0,7)$ & $(0,9)$ \\
\hline$(9,1)$ & $(0,2)$ & $(1,3)$ & $(2,4)$ & $(3,5)$ & $(2,3)$ & $(2,5)$ & $(2,9)$ & $(2,1)$ \\
\hline$(8,2)$ & $(9,3)$ & $(0,4)$ & $(1,5)$ & $(2,6)$ & $(4,5)$ & $(4,7)$ & $(4,1)$ & $(4,3)$ \\
\hline$(7,3)$ & $(8,4)$ & $(9,5)$ & $(0,6)$ & $(1,7)$ & $(6,7)$ & $(6,9)$ & $(6,3)$ & $(6,5)$ \\
\hline$(6,4)$ & $(7,5)$ & $(8,6)$ & $(9,7)$ & $(0,8)$ & $(8,9)$ & $(8,1)$ & $(8,5)$ & $(8,7)$ \\
\hline
\end{tabular}

Construct $B_{8}$ by deleting $f_{5}$ as well as vertices 0 and 5 and all edges connected to them.

Table 4. $B_{8}$

\begin{tabular}{|c|c|c|c|c|c|c|c|}
\hline$(1,6)$ & $(2,7)$ & $(3,8)$ & $(4,9)$ & $(2,3)$ & $(4,7)$ & $(2,9)$ & $(2,1)$ \\
\hline$(9,3)$ & $(1,3)$ & $(2,4)$ & $(2,6)$ & $(6,7)$ & $(6,9)$ & $(4,1)$ & $(4,3)$ \\
\hline$(8,4)$ & $(8,6)$ & $(9,7)$ & $(1,7)$ & $(8,9)$ & $(8,1)$ & $(6,3)$ & $(8,7)$ \\
\hline$p_{2}$ & $p_{4}$ & $p_{6}$ & $p_{8}$ & $p_{1}$ & $p_{3}$ & $p_{7}$ & $p_{9}$ \\
\hline
\end{tabular}


In this case, the edges are considered as information bits and $p_{i}$, for $1 \leq i \leq 9$ are considered as parity bits.

Construct extended B-Code of size $9, B_{9}$, from $B_{8}$ by adding a column of information bits. Those are the bits corresponding to the edges of $f_{5}$ which was deleted in construction of $B_{8}$.

\begin{tabular}{|c|c|c|c|c|c|c|c|c|}
\hline$(1,9)$ & $(1,6)$ & $(2,7)$ & $(3,8)$ & $(4,9)$ & $(2,3)$ & $(4,7)$ & $(2,9)$ & $(2,1)$ \\
\hline$(2,8)$ & $(9,3)$ & $(1,3)$ & $(2,4)$ & $(2,6)$ & $(6,7)$ & $(6,9)$ & $(4,1)$ & $(4,3)$ \\
\hline$(3,7)$ & $(8,4)$ & $(8,6)$ & $(9,7)$ & $(1,7)$ & $(8,9)$ & $(8,1)$ & $(6,3)$ & $(8,7)$ \\
\hline$(4,6)$ & $p_{2}$ & $p_{4}$ & $p_{6}$ & $p_{8}$ & $p_{1}$ & $p_{3}$ & $p_{7}$ & $p_{9}$ \\
\hline
\end{tabular}

The shortening from $B_{9}$ into $\tilde{X}_{5}$ can be obtained by first construct the shortening from $B_{8}$ into $\tilde{X}_{4}$. Set all information bits in the last 4 columns of array representation in $B_{8}$ to zero.

Table 6. $\tilde{X}_{4}$

\begin{tabular}{|c|c|c|c|c|c|c|c|}
\hline$(1,6)$ & $(2,7)$ & $(3,8)$ & $(4,9)$ & 0 & 0 & 0 & 0 \\
\hline$(9,3)$ & $(1,3)$ & $(2,4)$ & $(2,6)$ & 0 & 0 & 0 & 0 \\
\hline$(8,4)$ & $(8,6)$ & $(9,7)$ & $(1,7)$ & 0 & 0 & 0 & 0 \\
\hline$p_{2}$ & $p_{4}$ & $p_{6}$ & $p_{8}$ & $p_{1}$ & $p_{3}$ & $p_{7}$ & $p_{9}$ \\
\hline
\end{tabular}

The parities then can be written as:

$p_{1}=(1,6)+(1,3)+(1,7)$

$p_{3}=(9,3)+(1,3)+(3,8)$

$p_{7}=(2,7)+(9,7)+(1,7)$

$p_{9}=(9,3)+(9,7)+(4,9)$

which then can be rewritten as

$(1,6)=p_{1}+(1,3)+(1,7)$

$(3,8)=(9,3)+(1,3)+p_{3}$

$(2,7)=p_{7}+(9,7)+(1,7)$

$(4,9)=(9,3)+(9,7)+p_{9}$

Rename $(1,6),(3,8),(2,7),(4,9)$ as parities and $p_{1}, p_{3}, p_{7}, p_{9}$ as information bits, and set the information bits to zero as shown in table 7 .

Table 7. Zero information bits

\begin{tabular}{|c|c|c|c|c|c|c|c|}
\hline$p_{1}$ & $p_{7}$ & $p_{3}$ & $p_{9}$ & 0 & 0 & 0 & 0 \\
\hline$(9,3)$ & $(1,3)$ & $(2,4)$ & $(2,6)$ & 0 & 0 & 0 & 0 \\
\hline$(8,4)$ & $(8,6)$ & $(9,7)$ & $(1,7)$ & 0 & 0 & 0 & 0 \\
\hline$p_{2}$ & $p_{4}$ & $p_{6}$ & $p_{8}$ & 0 & 0 & 0 & 0 \\
\hline
\end{tabular}

Rearranging and removing the zeroed columns as shown in table 8.

\begin{tabular}{|cccc|}
\multicolumn{4}{|c}{ Table 8 . Rearranged information bits } \\
\hline$(9,3)$ & $(1,3)$ & $(2,4)$ & $(2,6)$ \\
$(8,4)$ & $(8,6)$ & $(9,7)$ & $(1,7)$ \\
\hline$p_{1}$ & $p_{7}$ & $p_{3}$ & $p_{9}$ \\
$p_{2}$ & $p_{4}$ & $p_{6}$ & $p_{8}$ \\
\hline
\end{tabular}

Adding the extra column in $B_{9}$ to this table will give the shortening $B_{9}$ into $\tilde{X}_{5}$

Table 9. $\tilde{X}_{5}$

\begin{tabular}{|c|cccc|}
\hline$(1,9)$ & $(9,3)$ & $(1,3)$ & $(2,4)$ & $(2,6)$ \\
$(2,8)$ & $(8,4)$ & $(8,6)$ & $(9,7)$ & $(1,7)$ \\
\cline { 2 - 5 }$(3,7)$ & $p_{1}$ & $p_{7}$ & $p_{3}$ & $p_{9}$ \\
$(4,6)$ & $p_{2}$ & $p_{4}$ & $p_{6}$ & $p_{8}$ \\
\hline
\end{tabular}

Separation of $\tilde{X}_{4}$ will give result as shown in table 10 and 11 .

Table 10. Separation of $\tilde{X}_{4}\left(p_{1}, p_{7}, p_{3}, p_{9}\right)$
\begin{tabular}{|cccc|}
\hline$(9,3)$ & $(1,3)$ & $(2,4)$ & $(2,6)$ \\
\hline$p_{1}$ & $p_{7}$ & $p_{3}$ & $p_{9}$ \\
\hline
\end{tabular}

Table 11. Separation of $\tilde{X}_{4}\left(p_{2}, p_{4}, p_{6}, p_{8}\right)$

\begin{tabular}{|cccc|}
\hline$(8,4)$ & $(8,6)$ & $(9,7)$ & $(1,7)$ \\
\hline$p_{2}$ & $p_{4}$ & $p_{6}$ & $p_{8}$ \\
\hline
\end{tabular}


And the separation of $\tilde{X}_{5}$ will give result as in table 12 and 13 .

Table 12. Separation of $\tilde{X}_{5}\left(p_{1}, p_{7}, p_{3}, p_{9}\right)$

\begin{tabular}{|c|cccc|}
\hline$(1,9)$ & $(9,3)$ & $(1,3)$ & $(2,4)$ & $(2,6)$ \\
\hline$(3,7)$ & $p_{1}$ & $p_{7}$ & $p_{3}$ & $p_{9}$ \\
\hline
\end{tabular}

Table 13. Separation of $\tilde{X}_{5}\left(p_{2}, p_{4}, p_{6}, p_{8}\right)$
\begin{tabular}{|c|cccc|}
\hline$(2,8)$ & $(8,4)$ & $(8,6)$ & $(9,7)$ & $(1,7)$ \\
\hline$(4,6)$ & $p_{2}$ & $p_{4}$ & $p_{6}$ & $p_{8}$ \\
\hline
\end{tabular}

These give extended two B-Codes of length $5, B_{5}$, with 4 parity bits, and the number of information bits is 6, i.e. the number of edges in $K_{4}$. In general Bohossian, Bruck, Wagner, and Xu [17] proved that the complete graph obtained from $B_{n}$ (separation from $\tilde{X}_{n}$ ) can give a perfect onefactorization of $K_{n+1}$.

\subsection{Disk Array Data Layout}

In [17], perfect one-factorization is modified to obtain a B-data layout. B-data layout is based upon the B-code explained in the first application above. The knowledge for this constructing is that disk space is logically structured as stripe units where each stripe unit consists of a fixed number of sectors. Each stripe unit will contain either client data or redundant data. Each code symbol in B-data layout designates a stripe unit and the array code is presented via the perfect onefactorization principle.

For example let $V=\{0,1,2, \ldots, p-1, \infty\}$ denote the vertex set. For $p>3$, the one-factor can be obtained as:

$$
P=\{(\infty, s),(s-1, s+1), \ldots,(s-n+1, s+n-1)\}
$$

With $\bmod p$ arithmetic.

The modification of perfect one-factorization is as follows:

(1) The factors are renamed $F_{i}$ for $1 \leq i \leq p-1$ so $F_{i}$ is the factor containing $(0, i)$. Each $F_{i}$ factor loses two edges, one incident on vertex 0 and one on vertex $\infty$.

(2) Each edge within modified factor $F_{i}$ specifies a parity value to be stored on disk drive $i$.

For example, consider $K_{7}$. This obviously has a perfect one-factorization.

The one-factor and the renamed factor $F_{i}$ can be listed as follows:

$P_{0}=\{(\infty, 0),(1,6),(2,5),(3,4)\}$

$P_{1}=\{(\infty, 1),(0,2),(3,6),(4,5)\}$

$F_{1}=\{(2,6),(3,5)\}$

$P_{2}=\{(\infty, 2),(1,3),(0,4),(5,6)\}$

$F_{2}=\{(3,6),(4,5)\}$

$P_{3}=\{(\infty, 3),(2,4),(1,5),(0,6)\}$

$F_{3}=\{(1,2),(4,6)\}$

$P_{4}=\{(\infty, 4),(3,5),(2,6),(1,0)\}$

$F_{4}=\{(1,3),(5,6)\}$

$P_{5}=\{(\infty, 5),(4,6),(3,0),(2,1)\}$

$F_{5}=\{(1,4),(2,3)\}$

$P_{6}=\{(\infty, 6),(5,0),(4,1),(3,2)\}$

$F_{6}=\{(1,5),(2,4)\}$

Then $\mathrm{B}_{6}$ data layouts can be obtained as shown in table 14 .

Table 14. $\mathrm{B}_{6}$

\begin{tabular}{|c|c|c|c|c|c|}
\hline disk 1 & disk 2 & disk 3 & disk 4 & disk 5 & disk 6 \\
\hline$d_{1}$ & $d_{2}$ & $d_{3}$ & $d_{4}$ & $d_{5}$ & $d_{6}$ \\
\hline$d_{2} \oplus d_{6}$ & $d_{3} \oplus d_{6}$ & $d_{1} \oplus d_{2}$ & $d_{1} \oplus d_{3}$ & $d_{1} \oplus d_{4}$ & $d_{1} \oplus d_{5}$ \\
\hline$d_{3} \oplus d_{5}$ & $d_{4} \oplus d_{5}$ & $d_{4} \oplus d_{6}$ & $d_{5} \oplus d_{6}$ & $d_{2} \oplus d_{3}$ & $d_{2} \oplus d_{4}$ \\
\hline
\end{tabular}

where $\oplus$ denotes exclusive-OR operation.

As it is explained in two examples above, perfect one-factorization is well known for its use in coding and cryptography, especially in problem related to MDS array code.

\section{Conclusion}

Since it was introduced in 1964, there has been a great improvement in research of proving the existence of Perfect One-Factorization for complete graph $K_{2 n}$, for every integer $n \geq 2$. Although the exact and general result have not been accomplished yet, the studies conducted have shown that the work for this has almost reached the result. Many studies were based on various methods and basic knowledge, for example basic and abstract algebra and combinatorics. Regarding the results 
obtained so far, it is not impossible to conclude that the conjecture might exist. Furthermore, considering the applications, the perfect one-factorization can still be used in many fields.

\section{References}

[1] Kotzig, A., 1964. Hamilton Graphs and Hamilton Circuits, In : Theory of Graphs and Its Applications, Publ. House Czechoslovak Acad. Sci., Prague.

[2] Anderson, B., 1973. Finite Topologies and Hamiltonian Paths, Journal of Combinatorial Theory Series, B, 14:87-93.

[3] Nakamura, G., 1975. Dudeney's Round Table Problem and the Edge-colouring of the Complete Graphs (in Japanese), Proceeding in Sugaku Seminar, 159:24-29.

[4] Kobayashi, M., 1989. On Perfect One-factorization of the Complete Graph $\mathrm{K}_{2 \mathrm{p}}$, Graphs and Combinatorics, 5:351-353.

[5] Byant, D., B. Maenhaut and I. Wanless, 2006. New Families of Atomic Latin Squares and Perfect 1-factorisations, Journal Combinatorial Theory Series A, 113:608-624.

[6] Wagner, D., 1992. On the Perfect One-factorization Conjecture, Discrete Mathematics, 104:211-215.

[7] Wallis, W., 2007. A Beginner's to Graph Theory $2^{\text {nd }}$ edition. Birkhäuser Boston Publisher, Boston.

[8] Dinitz, J. and D. Stinson, 1989. Some New Perfect One-factorizations from Starter in Finite Fields, Journal of Graph Theory, 13:405-415.

[9] Dinitz, J. and P. Dukes, 2006. On the Structure of Uniform One-factorizations from Starters in Finite Fields, Finite Fields and Their Applications, 12:283-300.

[10] Anderson, B. and D. Morse, 1974. Some Observations on Starters, Proceeding of $5^{\text {th }}$ Southeastern Conf. on Combinatorics, Graph Theory and Computing, Congressus Numeratium, 229-235.

[11] Seah, E. and D. Stinson, 1988. A Perfect One-factorization for $\mathrm{K}_{36}$, Discrete Mathematics, 70: 199-202.

[12] Seah, E. and D. Stinson, 1989. A Perfect One-factorization for $\mathrm{K}_{40}$, Congressus Numeratium, 68:211-213.

[13] Ihrig, E., E. Seah and D. Stinson, 1987. A Perfect One-factorization of $\mathrm{K}_{50}$, Journal of Combinatorial Mathematics and Combinatorial Computing, 1:217-219.

[14] Kobayashi, M., and Kiyasu-Zen'iti, 1989. Perfect One-factorizations of $\mathrm{K}_{1332}$ and $\mathrm{K}_{6860}$, Journal of Combinatorial Theory Series A, 51:314-315.

[15] Wanless, I., 2014. http://users.monash.edu.au/ iwanless/data/P1F/newP1F.html.

[16] Bohossian, V., and J. Bruck, 2006. Shortening Array Codes and the Perfect 1-factorization conjecture, IEEE International Symposium on Information Theory, 2799-2803.

[17] Bohossian, V., J. Bruck, D. Wagner and L. Xu, 1999. Low-density MDS Codes and Factors of Complete Graphs, IEEE-Trans-IT, 45:1817-1826. 